\title{
A Simulation Study on the Impact of Activity Crashing on the Project Duration and Cost under Different Budget Release Scenarios
}

\author{
Jie Song*, Tom Servranckx*, Annelies Martens* and Mario Vanhoucke* ${ }^{* \dagger}$ \\ ${ }^{*}$ Faculty of Economics and Business Administration \\ Ghent University, Tweekerkenstraat 2, 9000 Gent, Belgium \\ Email: jieson.song@ugent.be \\ ${ }^{\dagger}$ Technology and Operations Management Area, Vlerick Business School, Reep 1, 9000 Gent, Belgium \\ $\ddagger$ UCL School of Management, University College London, 1 Canada Square, London E14 5AA, UK
}

\begin{abstract}
The main goal of project control is to identify project opportunities or problems during project execution, such that corrective actions can be taken to bring the project in danger back on track when necessary. In this study, we define different scenarios to allocate the limited budget used for the cost of activity execution, delays, and corrective actions, according to the timing and amount of the budget release. A large computational experiment is conducted on real-life project data to evaluate the performance of each scenario. The results show that both the timing and amount of the budget release have an effect on project performance.
\end{abstract}

\section{INTRODUCTION}

$\mathbf{P}$ ROJECT control is a key part of project management $(\mathrm{PM})$, together with baseline scheduling and schedule risk analysis. Where baseline scheduling focuses on the construction of a timetable for the activities considering the technological and resource constraints in the project, risk analysis identifies high risk activities in the project. Both aspects belong to the static PM phase, i.e. prior to project execution, and are supported by state-of-the-art optimisation and simulation techniques to create optimal and robust project schedules. In contrast, project control is the process of monitoring the project during execution to detect potential problems and taking corrective actions when necessary, and belongs to the dynamic PM phase [1]. Since project success can only be achieved when the static PM phase is combined with an effective project control process, advanced modelling and simulation techniques are needed to support the project control process.

Earned Value Management (EVM) is a project control method to measure the project performance in terms of time and cost [2]. Using EVM, the project progress can be periodically measured and compared to a control limit [3]. When the progress is below this limit, the project is expected to exceed its deadline and a warning signal is generated to initiate corrective action. Since generating efficient and reliable warning signals is important to take effective corrective actions, this research topic has been investigated intensively in recent years [4], [5], [6]. Other recent research studies focused on the corrective action taking process [7], [8], [9]. Since many of these research efforts do not consider the fact that the project budget is limited in most real-life projects, [10] have investigated the performance of four different approaches to allocate a limited budget dedicated to corrective actions over different project phases. The authors developed an extensive simulation experiment to evaluate the four approaches using a large set of artificial projects and showed that the best allocation model considers the planned progression of work in the project and provides a control budget that increases in later project stages.

In this study, we simulate the impact of time-cost tradeoffs given a limited project budget on the time and cost performance of a set of real-life projects. The timing and quantity of the release of the budget throughout the project life cycle is modelled using different scenarios: immediate or time-phased budget releases, proportional to the time or cost profile of the project and dynamically increasing or decreasing as the project progresses. We only consider activity crashing as a potential corrective action, which implies investing more budget in an activity to reduce the activity duration (i.e. time-cost trade-offs). This problem is related to the Project Scheduling Game (PSG, [11]), a project control game in which the project execution of a relatively complex project is simulated. The objective is to minimise the final project cost by controlling the project at six decision moments using activity crashing. While an unlimited budget is available in the PSG, we evaluate different strategies for using a limited budget during project execution to complete a project within this budget. Further, we extend the existing research by [10] in three ways. First, we do not consider a control budget that can only be used for activity crashing, but we determine a total project budget that should cover the cost of activity execution, delays and activity crashing. This is a more realistic situation since the cost of activity delays and penalty costs for project delays are explicitly considered. Second, our focus is on cost minimisation rather than timely project completion. Therefore, we consider projects that exceed the predefined project budget to be failed projects and we evaluate the performance of the different scenarios based on the actual project duration 
and cost and the portion of failed projects. Finally, we test the proposed approaches on real-life project data in order to incorporate realistic cost profiles.

\section{Methodology}

The methodology of this study consists of four phases. In the data collection phase, planning and risk data from real-life projects are collected. In the scenario analysis phase, different scenarios to allocate the project budget over the project life cycle are defined. During the simulation phase, progress data for each of the real-life projects is simulated to review the impact of activity crashing on the cost profile of the projects. The evaluation phase consists of an analysis of the duration and cost performance of the projects. Table I summarises the relevant terminology and parameters used in this study.

\section{A. Data collection}

In the data collection phase, real-life planning, progress and risk data from projects in various industries has been collected and documented. After a first meeting, the project owner or manager decides whether they are willing to collaborate. If this is the case, a project that is planned to start in the near future is selected for real-time periodical follow-up. This ensures that the documented data is correct and complete. At each period, it is reviewed whether the information is available at the activity level. If activity level data is available, the activity risk profiles are discussed with the project owner or manager. Otherwise, the follow-up process is terminated. When all collected data is clear, it is registered in a structured manner. This process is repeated periodically until the project is finished.

Table II gives an overview of the collected data and lists the industry, planned duration (PD), Budget at Completion (BAC) and number of activities (nract) of each project. These projects are included in the database of [12] and are online available at https://www.projectmanagement.ugent.be/research/data/realdata.

\section{B. Scenario analysis}

Before the project start, the total project budget is defined as the BAC (section II-A) increased with a management reserve to take corrective actions and to deal with activity delays during execution. In the computational experiment, the size of the management reserve is varied and different scenarios considering the timing and quantity of the budget release are considered for the release of the project budget during execution.

a) Size of management reserve: In this study, the size of the management reserve (MR) is defined as the difference between the average actual project cost (APC) without activity crashing and the BAC, multiplied with a factor $m_{B}$ (equation (1)). The APC without activity crashing is calculated by adding uncertainty profiles to the activity durations and using Monte Carlo simulations to imitate the actual project progress (section II-C).

$$
\mathrm{MR}=(\mathrm{APC}-\mathrm{BAC}) \times m_{B}
$$

b) Timing of budget release: Two different approaches are considered regarding the timing of the budget release. First, the immediate approach assumes that the entire project budget is made available from the start of the project. Second, the time phased approach assumes that the project is divided in different phases, and a portion of the total project budget is released at the start of each phase.

c) Quantity of budget release: The quantity of the budget release depends on the applied timing approach. For the immediate timing approach, the entire budget $(\mathrm{BAC}+$ management reserve) is released at the start of the project. For the time phased approach, the planned budget at the end of each phase is increased with a portion of the management reserve and released at the start of each phase. To determine the portion of the management reserve to be released at each phase, two viewpoints are used. First, using the time focus viewpoint (equation (2)), the management reserve is allocated to each phase proportional with the relative duration of each phase $\left(\frac{\mathrm{PD}_{\text {phase }}}{\mathrm{PD}_{\text {project }}}\right)$. Second, using the cost focus viewpoint (equation (3)), the management is allocated to each phase proportional with the relative budgeted cost of each phase $\left(\frac{B A C_{\text {phase }}}{B A C_{\text {project }}}\right)$.

$$
\begin{aligned}
& \text { Assigned budget }_{\text {phase, time }}=\mathrm{BAC}_{\text {phase }}+\frac{\mathrm{PD}_{\text {phase }}}{\mathrm{PD}_{\text {project }}} \times \mathrm{MR} \\
& \text { Assigned budget }_{\text {phase, cost }}=\mathrm{BAC}_{\text {phase }}+\frac{\mathrm{BAC}_{\text {phase }}}{\mathrm{BAC}_{\text {project }}} \times \mathrm{MR}
\end{aligned}
$$

While equations (2) and (3) allocate the management reserve over the different phases proportionally with the time or cost (i.e. the standard version), the management reserve can be allocated in a dynamically increasing or decreasing way as well [10]. The increasing version ensures that the allocated management reserve is relatively low at the start of the project and systematically increases along the project progress by allocating the management reserve based on the square of the relative duration or cost of each phase (Equation (4)). The decreasing version uses the square root to start with a relatively high amount of the budget which increases degressively along the project progress (Equation 5). In table III, an overview of the scenarios reviewed in the simulation experiment is given.

Assigned budget $_{\text {phase, time, increasing }}=\mathrm{BAC}_{\text {phase }}+\left(\frac{\mathrm{PD}_{\text {phase }}}{\mathrm{PD}_{\text {project }}}\right)^{2} \times \mathrm{MR}$
Assigned budget
phase, time, decreasing

\section{Simulation}

In the simulation phase, 1,000 simulated project executions are generated for each project collected in the data collection phase. Before the start of each project, the tolerance limits for the project progress are set as introduced by [4]. During each simulated execution, the project progress is measured and compared to these limits periodically. When the incurred 
TABLE I

OVERVIEW OF TERMINOLOGY

\begin{tabular}{|c|c|c|c|c|c|}
\hline \multirow{2}{*}{$\begin{array}{l}\text { Concepts } \\
\text { Project }\end{array}$} & & \multicolumn{2}{|c|}{ Parameters } & \multicolumn{2}{|c|}{ Performance measures } \\
\hline & number of activities & $\begin{array}{l}\text { Project } \\
m_{B}\end{array}$ & multiplier total budget & AFP & Actual Failed Projects \\
\hline BAC & Budget at Completion & $m_{D}$ & multiplier delay cost & APD & Actual Project Duration \\
\hline PD & Planned Duration & $C_{D}$ & Cost of unit delay & APC & Actual Project Cost \\
\hline $\mathrm{AD}$ & Actual Duration & & $=\frac{\mathrm{BAC}}{\mathrm{PD}} \times m_{D}$ & & \\
\hline Activities & & Activities & & & \\
\hline $\begin{array}{l}\mathrm{AD}_{i} \\
C_{E_{i}}\end{array}$ & $\begin{array}{l}\text { Actual duration act } i \\
\text { Fixed cost of act } i\end{array}$ & $C_{C, i}$ & $\begin{array}{l}\text { Crash cost of act } i \\
=2 \times C_{F}\end{array}$ & & \\
\hline$C_{V, i}^{F, i}$ & Variable cost of act $i$ & & & & \\
\hline $\mathrm{UC}_{i}$ & Crashed units of act $i$ & & & & \\
\hline
\end{tabular}

TABLE II

OVERVIEW OF REAL-LIFE PROJECTS

\begin{tabular}{ccccc} 
Project ID & Industry & PD (days) & BAC $(€)$ & nract \\
\hline C2011_03 & Event & 97 & 31,675 & 24 \\
C2011_04 & Construction & 125 & 59,831 & 20 \\
C2011_05 & Telecom & 43 & 180,485 & 23 \\
C2011_08 & Construction & 72 & 254,564 & 28 \\
C2011_11 & Event & 299 & 37,760 & 26 \\
C2012_01 & Manufacturing & 45 & 61,699 & 31 \\
C2012_11 & Manufacturing & 13 & $1,535,854$ & 24 \\
C2013_17 & Construction & 161 & 244,205 & 25 \\
C2014_07 & Construction & 353 & $1,102,537$ & 27 \\
C2014_08 & Construction & 233 & $1,992,222$ & 41
\end{tabular}

costs at the period exceed the released budget until that time, the project is interrupted until an additional part of the project budget is released and the project can be resumed. Further, when the total project budget is exceeded, the project is terminated and classified as a failed project. When the project is not interrupted or terminated, the project progress is reviewed. If the progress is below the tolerance limit, the activities eligible for activity crashing are determined by comparing the activity crash cost of the ongoing critical activities (i.e. activities on the critical path) to the expected delay cost reduction. If there are eligible activities and the required budget for activity crashing is available at the period, the actions are taken and the project is continued.

\section{Performance evaluation}

After completion of the simulation phase, the performance of the simulated executions is reviewed. For each simulation experiment, the number of failed projects (AFP), i.e. the number of projects that exceeded their budget, is observed. Further, the time and cost performance is evaluated using the actual project duration (APD) and actual project cost (APC). The APD and APC are expressed relatively to the planned duration and the total project budget of the projects, respectively.

\section{RESULTS AND DISCUSSION}

In this section, the performance of the scenarios depicted in table III is evaluated using the project performance measures listed in table I (Experiment 1). In Experiment 2, the impact on the project performance of a change in the total project budget is examined by varying the value of $m_{B}$ (equation (1)). Finally, Experiment 3 reviews the impact of changes in the cost per unit delay $\left(C_{D}=\frac{\mathrm{BAC}}{\mathrm{PD}} \times m_{D}\right)$ by varying the value of $m_{D}$.

a) Experiment 1: Comparison of scenarios: The results of Experiment 1 are summarised in table IV. The results show that the immediate budget release approach (S1) outperforms the time phased budget release approaches in terms of AFP, APD and APC. All project runs are finished within the assigned project budget, with an average duration of $101.7 \%$ of the PD. Further, for the standard time phased assignment versions, the time focus (S2) performs better than the cost focus (S5) for all performance measures. This can be explained by the fact that the cost focus assigns higher portions of the management reserve to more costly project phases. Since the activities planned in these phases are typically more expensive to crash, less corrective actions can be taken with the same budget. Finally, the decreasing version of the time focus approach (S3) uses the available project budget most effectively, since it results in the lowest AFP, APD and APC of all time phased approaches.

b) Experiment 2: Impact of changes in total project budget size: Since experiment 1 showed that the time phased scenarios using a time focus outperform the scenarios using a cost focus, the remaining discussion focuses on scenarios S1-S4. In general, table V shows that reducing the size of the management reserve increases the APD, APC and especially the AFP. For an immediate budget release (S1), a reduction of $10 \%\left(m_{B}=0.9\right)$ has a limited impact, while a reduction of $20 \%\left(m_{B}=0.8\right)$ results in considerably more failed projects. For the time phased scenarios, the impact of reducing the budget is more substantial. For a reduction of $10 \%, \mathrm{~S} 3$ still outperforms S2 and S4. When the management reserve is reduced with $20 \%$, however, the performance of these scenarios become comparable.

c) Experiment 3: Impact of changes in the cost of delays: Table VI shows that reducing the unit cost of delay $\left(m_{D}\right)$ has a limited impact. Both the AFD, APD and APC increase slightly for lower unit costs of delay. The increase in APC can be explained by the fact that activities are only crashed when the expected reduction in delay costs is higher than the increased costs due to the crashing action.

To conclude, this simulation experiment indicates that a management reserve should be considered to control projects during execution. Experiment 1 shows that both the timing 
TABLE III

OVERVIEW OF CONSIDERED SCENARIO SETTINGS

\begin{tabular}{l|ccccccc} 
Scenario & S1 & S2 & S3 & S4 & S5 & S6 & S7 \\
\hline Timing & Immediate & Time phased & Time phased & Time phased & Time phased & Time phased & Time phased \\
Quantity & - & Time & Time & Time & Cost & Cost & Cost \\
Version & - & Standard & Decreasing & Increasing & Standard & Decreasing & Increasing
\end{tabular}

TABLE VI

IMPACT OF CHANGES IN COST OF DELAYS $\left(m_{B}=1\right)$

\begin{tabular}{ccccc} 
Scenario & $\boldsymbol{m}_{\boldsymbol{D}}$ & AFP $(\boldsymbol{\%})$ & APD $(\boldsymbol{\%})$ & APC $(\boldsymbol{\%})$ \\
\hline \multirow{3}{*}{ S1 } & $\mathbf{1 . 0 0}$ & 0 & 101.7 & 89.6 \\
& $\mathbf{0 . 7 5}$ & 0 & 101.7 & 91.1 \\
& $\mathbf{0 . 5 0}$ & 0 & 101.7 & 92.6 \\
S2 & $\mathbf{1 . 0 0}$ & 16 & 105.4 & 90.8 \\
& $\mathbf{0 . 7 5}$ & 20 & 106.4 & 92.5 \\
& $\mathbf{0 . 5 0}$ & 22 & 107.1 & 92.6 \\
S3 & $\mathbf{1 . 0 0}$ & 6 & 102.9 & 89.7 \\
& $\mathbf{0 . 7 5}$ & 8 & 103.3 & 91.2 \\
& $\mathbf{0 . 5 0}$ & 11 & 103.9 & 92.8 \\
S4 & $\mathbf{1 . 0 0}$ & 28 & 108.5 & 92.7 \\
& $\mathbf{0 . 7 5}$ & 32 & 109.1 & 93.7 \\
& $\mathbf{0 . 5 0}$ & 38 & 109.7 & 94.6
\end{tabular}

TABLE IV

COMPARISON OF SCENARIOS $\left(m_{B}=1, m_{D}=1\right)$

\begin{tabular}{cccc} 
Scenario & AFP $(\boldsymbol{\%})$ & APD $(\boldsymbol{\%})$ & APC $(\boldsymbol{\%})$ \\
\hline S1 & 0.0 & 101.7 & 89.6 \\
S2 & 15.8 & 105.4 & 90.8 \\
S3 & 5.8 & 102.9 & 89.7 \\
S4 & 27.7 & 108.5 & 92.7 \\
S5 & 19.8 & 105.8 & 91.2 \\
S6 & 6.1 & 103.1 & 89.7 \\
S7 & 33.7 & 111.3 & 94.4
\end{tabular}

TABLE V

IMPACT OF CHANGES IN TOTAL PROJECT BUDGET SIZE $\left(m_{D}=1\right)$

\begin{tabular}{ccccc} 
Scenario & $\boldsymbol{m}_{\boldsymbol{B}}$ & AFP $(\boldsymbol{\%})$ & APD $(\boldsymbol{\%})$ & APC $(\boldsymbol{\%})$ \\
\hline \multirow{3}{*}{ S1 } & $\mathbf{1 . 0}$ & 0 & 101.7 & 89.6 \\
& $\mathbf{0 . 9}$ & 3 & 101.8 & 91.5 \\
& $\mathbf{0 . 8}$ & 19 & 102.7 & 94.0 \\
S2 & $\mathbf{1 . 0}$ & 16 & 105.4 & 90.8 \\
& $\mathbf{0 . 9}$ & 41 & 108.7 & 94.6 \\
& $\mathbf{0 . 8}$ & 72 & 113.3 & 99.7 \\
S3 & $\mathbf{1 . 0}$ & 6 & 102.9 & 89.7 \\
& $\mathbf{0 . 9}$ & 28 & 105.8 & 92.7 \\
& $\mathbf{0 . 8}$ & 70 & 111.8 & 98.6 \\
S4 & $\mathbf{1 . 0}$ & 28 & 108.5 & 92.7 \\
& $\mathbf{0 . 9}$ & 50 & 110.7 & 95.9 \\
& $\mathbf{0 . 8}$ & 75 & 114.0 & 100.2
\end{tabular}

and amount of the budget release have an effect on the actual project duration and cost. Further, experiment 2 shows that the total size of the management reserve is of importance as well. If the management reserve is too low, the performance of different strategies for the amount of budget release perform equally low. Finally, when the cost of delays is decreased, this has a more substantial impact on the actual project cost than on the actual project duration.

\section{REFERENCES}

1] M. Vanhoucke, Project Management with Dynamic Scheduling: Baseline Scheduling, Risk Analysis and Project Control. Springer, 2012, vol. XVIII.

2] Q. Fleming and J. Koppelman, Earned Value Project Management, 3rd ed. Newton Square, Pennsylvania: Project Management Institute, 2010. [Online]. Available: http://books.google.be/books? id=ZMRVngEACAAJ

[3] J. Colin and M. Vanhoucke, "Setting tolerance limits for statistical project control using earned value management," Omega The International Journal of Management Science, vol. 49, pp. 107-122, 2014.

[4] A. Martens and M. Vanhoucke, "A buffer control method for top-down project control," European Journal Of Operational Research, vol. 262, pp. 274-286, 2017.

[5] J. Zhang, S. Jia, and E. Diaz, "Dynamic monitoring and control of a critical chain project based on phase buffer allocation," Journal of the Operational Research Society, vol. 69, pp. 1-12, 2018.

[6] H. Hadian and A. Rahimifard, "Multivariate statistical control chart and process capability indices for simultaneous monitoring of project duration and cost," Computers \& Industrial Engineering, pp. 788-797, 2019.

[7] M. Madadi and H. Iranmanesh, "A management oriented approach to reduce a project duration and its risk (variability)," European Journal of Operational Research, vol. 219, no. 3, pp. 751-761, 2012.

[8] P. Ballesteros-Pérez, K. Elamrousy, and M. Gonz'ales-Cruz, "Nonlinear time-cost trade-off models of activity crashing: Application to construction scheduling and project compression with fast-tracking," Automation in Construction, vol. 97, pp. 229-240, 2019.

[9] A. Martens and M. Vanhoucke, "The impact of applying effort to reduce activity uncertainty on the project time and cost performance," European Journal of Operational Research, vol. 277, no. 2, pp. 442-453, 2019.

[10] J. Song, A. Martens, and M. Vanhoucke, "The impact of a limited budget on the corrective action taking process," European Journal Of Operational Research, vol. 286, no. 3, pp. 1070-1086, 2020.

[11] M. Vanhoucke, A. Vereecke, and P. Gemmel, "The project scheduling game (PSG): Simulating time/cost trade-offs in projects," Project Management Journal, vol. 51, pp. 51-59, 2005.

[12] J. Batselier and M. Vanhoucke, "Construction and evaluation framework for a real-life project database," International Journal of Project Management, vol. 33, pp. 697-710, 2015 